\title{
Wavelet-based analysis of low-frequency fluctuations of blood pressure and sympathetic nerve activity in rats
}

\author{
Meng-Li Tsai ${ }^{\mathrm{a} *}$, Wei-Chang Shann ${ }^{\mathrm{b}}$, Wen-Ren Luo ${ }^{\mathrm{b}}$, Chen-Tung Yen $^{\mathrm{c}}$ \\ ${ }^{a}$ Department of Biomachtronic Engineering, National Ilan University, No. 1 Shen Lung Rd., Sect. 1, Ilan 260, Taiwan \\ ${ }^{\mathrm{b}}$ Department of Mathematics, National Central University, Taoyuan, Taiwan \\ ${ }^{\mathrm{c}}$ Institute of Zoology and Department of Life Science, National Taiwan University, Taipei, Taiwan
}

Received 22 December 2003; received in revised form 8 January 2004; accepted 10 January 2004

\begin{abstract}
Biorthogonal wavelets were employed to quantify the relationship of fluctuations between blood pressure (BP) and sympathetic nerve activity (SNA). We forced the SNA to fluctuate by electrical stimulation the medulla in anesthetized, paralyzed, vagotomized, cardiac sympathetic-blocked, baroreceptor-denervated, and angiotensin II-converting enzyme-inhibited rats. Although spectral analysis showed a close coupling between fluctuations of BP and SNA at the stimulating frequencies, only the fluctuations of SNA in frequencies of $0.25 \sim 0.4$ $\mathrm{Hz}$ were proportional to BP fluctuations over the course of time. The results suggest that fluctuations transmitted from SNA to BP were uniform without shifting due to the nature of vasculature or the lagging of sympathetic action in frequencies of $0.25 \sim 0.4 \mathrm{~Hz}$, and support the possibility of using low-frequency variabilities of BP to quantitatively estimate fluctuations of SNA at time domain.

(C) 2004 Elsevier Ireland Ltd. All rights reserved.
\end{abstract}

Keywords: Wavelet; Blood pressure; Sympathetic nerve activity; Low frequency fluctuation; Fast Fourier transformation; Transfer function

Sympathetic nerve activity (SNA) is an important parameter in certain physiological and pathophysiological situations. Therefore, a reliable, non-invasive method to measure SNA would be an important diagnostic tool for both basic and clinic research. Several lines of evidence have suggested that sympathetic nerve activity is a critical determinant of blood pressure (BP) fluctuations at a frequency range which is slower than the respiration rate [5], and the strength of the lowfrequency BP variability is presumed to represent proportional changes in vascular sympathetic outflow [10]. However, controversies still remain. For example, findings in animals suggest that low-frequency fluctuations of BP do not reflect sympathetic outflows [12], but instead are related to the level of sympathetic oscillations $[9,15]$. Furthermore, a consensus on the range of the low-frequency band has not been established, the following frequency bands have all been suggested for rats: $0.1 \sim 0.18 \mathrm{~Hz}$ [2], $0.27 \sim 0.74 \mathrm{~Hz}$ [7], $0.38 \sim 0.45 \mathrm{~Hz}[4], 0.3 \sim 0.6 \mathrm{~Hz}[6], 0.25 \sim 0.75 \mathrm{~Hz}[8]$, $0.2 \sim 0.8 \mathrm{~Hz}[12], 0.016 \sim 0.85[15]$ and exactly at $0.4 \mathrm{~Hz}[3]$.

\footnotetext{
* Corresponding author. Tel.: +886-3-935-7400 ext. 851; fax: + 886-3932-6345.

E-mail address: mltsai@niu.edu.tw (M.L. Tsai).
}

Different mathematical methods have been used to analyze the variability of BP or SNA. Among these, fast Fourier transformation (FFT) is the one most commonly chosen [13]. However, FFT has its own drawbacks and limitations. The main problem is that the FFT-based spectral estimation assumes that the analyzed signal is stationary. For its averaging process, the power coefficients at a given frequency resulting from that analysis are a mixture of events occurring at different times. To overcome the limitations, wavelet analysis methods have been used in the signal processing of biomedical signals [1]. The methods represent temporal characteristics of a signal with its spectral components in the frequency domain. These are a powerful alternative for the analysis of non-stationary signals whose spectral features are changing over the course of time. We assess the relationship between fluctuations in SNA and BP with discrete wavelet transformation (DWT) in this study. Two questions are investigated: first, the frequency band which can be used as an quantitative index of sympathetic modulation in time, and second, the manner of indexing.

Three male Wistar rats $(380 \sim 450 \mathrm{~g})$ were used. The entire experimental procedure was approved by the 
Institutional Animal Care and Use Committee, National Ilan University. Details of surgical procedures were the same as those in the previous study [14]. Briefly, each rat was anesthetized with sodium pentobarbital $(50 \mathrm{mg} / \mathrm{kg}$, i.p. initially and $12.5 \mathrm{mg} / \mathrm{kg}$ i.v., supplemented as necessary during surgery and every $30 \mathrm{~min}$ during the recording period). The femoral artery and vein and trachea were cannulated for systemic BP recording, drug administration, and positive pressure ventilation, respectively. The endexpiratory $\mathrm{CO}_{2}$ concentration was maintained at between 3.5 and $4.0 \%$ and rectal temperature was maintained at $37.5 \pm 0.1^{\circ} \mathrm{C}$. The left renal nerve was dissected free from the surrounding connective tissues and crushed distally. A bipolar hook electrode made of stainless steel microwires (biomedical wire AS 634, Cooner Wire; Chatsworth, CA) was positioned under the renal nerve. Both the exposed nerve and electrode were encased in silicon gel (Wacker Siligel 604; Munich, Germany). Baroreceptor denervation was performed by severing the carotid sinus nerves, glossopharyngeal nerves, superior laryngeal nerves, and sympathetic trunks near the superior cervical ganglia. The carotid bifurcations were stripped of fibers, and connective tissues and both vagi were cut near their entrance into the skull.

The head of the rat was fixed in a stereotaxic apparatus in a prone position with the bite bar $10 \mathrm{~mm}$ below the interaural line. Gallamine $(50 \mathrm{mg} / \mathrm{kg})$, atenolol $(1 \mathrm{mg} / \mathrm{kg})$, and captopril $(10 \mathrm{mg} / \mathrm{kg})$ were i.v. injected. Supplementary dosages were administered every $10 \sim 15 \mathrm{~min}$ in the amounts of one-fourth of the initial dosage. A bipolar stimulation electrode was lowered into the medullary oblongata. The area stimulated included a cubic space which included an area $2.0 \mathrm{~mm}$ rostral to and $2.0 \mathrm{~mm}$ caudal to the obex, $0 \sim 2.0 \mathrm{~mm}$ lateral to the midline, and $0.5 \sim 2.5 \mathrm{~mm}$ dorsal to the ventral surface of the medulla. When a sympathoexcitatory point was found, trains of electrical pulses were used to activate the sympathetic nervous system (Anapulse stimulator model 302-T, WPI, New Haven, CT). Parameters of the stimulation were a pulse duration of $500 \mu \mathrm{s}$, pulse intensity of $10 \sim 50 \mu \mathrm{A}$, pulse frequency of $50 \mathrm{~Hz}$, and train duration of $500 \mathrm{~ms}$. The inter-train interval varied from 0.6 to $50 \mathrm{~s}$. The total period of stimulation was $80 \sim 90 \mathrm{~s}$. When the blood pressure and renal nerve activity had returned to a steady state, the stimulation was repeated. After the last stimulation-recording period, hexamethonium bromide (20 mg/kg, i.v.) was given to determine the baseline value of renal nerve activity.

The BP signal was obtained through a pressure-voltage transducer (Gould P23 ID) and amplified (Grass 7D). The electrical signal of the renal nerve was band pass-filtered between $30 \sim 3 \mathrm{KHz}$ and amplified 10,000 times by a Grass P511 differential amplifier. All signals were stored on a tape recorder (model DR-886, Neuro Data, New York, NY). For off-line analysis, a 64-s analysis period was used. Signals were digitized at $6 \mathrm{~K}$ samples/s using an MP $100 \mathrm{~A} / \mathrm{D}$ converter (BIOPAC System, Goleta, CA). Renal nerve signals were integrated using a digital integrator with a 20$\mathrm{ms}$ reset time. The digitized BP and integrated SNA signals were resampled at $128 \mathrm{~Hz}$.

We chose $(6,8)$ biorthogonal wavelets for this study because their coefficients are readily available in the Matlab Wavelet Toolbox. DWT was applied as a filter to obtain the signal at the 8th or 9th scale and to analyze both BP and SNA signals at this scale. All computations in this study were performed by Matlab 6.5 running on an Intel PC. The wavelet data within each order were normalized as $\mathrm{Z}$ values, i.e. scaled by the standard deviation of activities during the analyzed period after subtracting the mean values of the activities during the analyzed period. The power densities of each frequency in the FFT analysis were normalized by dividing by the averaged power within the frequency band of $0 \sim 0.85 \mathrm{~Hz}$. The transfer function, $\mathrm{H}(\mathrm{f})$, was calculated as $\mathrm{H}(\mathrm{f})=\mathrm{P}_{\mathrm{BP}} / \mathrm{P}_{\mathrm{SNA}}$, where $\mathrm{P}_{\mathrm{BP}}$ and $\mathrm{P}_{\mathrm{SNA}}$ are the respective normalized power densities of BP and SNA at each frequency.

Examples of integrated SNA and the corresponding fluctuations in BP during different stimulation periods are shown in Fig. 1BI. A close coupling between BP and SNA spectral peaks was observed at low-frequency stimulations (Fig. 1BII) as described in a previous study [14]. DWT was further applied to BP and SNA signals. The multi-resolution decomposition separates the signal into 'details' and 'approximations' at different scales (Fig. 1A). Only the details or the approximation contain one peak of sympathetic fluctuation within its frequency range which was selected for further analysis (Fig. 1BIII). The BP and SNA signals analyzed by wavelet transformation and $\mathrm{Z}$ scale normalization demonstrated tighter coupling and a better linear fit at stimulating frequencies lower than $0.5 \mathrm{~Hz}$ (Fig. 1BIV). Linear regression analysis of the relationship between the wavelet-transformed BP and SNA of all stimulation epochs in the three rats demonstrated that the frequency band of $0.25 \sim 0.4 \mathrm{~Hz}$ shows higher correlation than the other frequency ranges (Fig. 2A). Pooling all of the paired BP and SNA wavelet-transformed data within the frequency band of $0.25 \sim 0.4 \mathrm{~Hz}$ of the three rats also showed a high linear fit ( $r=0.74$, Fig. 2B), and a negative proportional relationship was obtained $\left(\mathrm{W}_{\mathrm{SNA}}=-0.75\right.$ $\mathrm{W}_{\mathrm{BP}} ; \mathrm{W}_{\mathrm{BP}}$ and $\mathrm{W}_{\mathrm{SNA}}$ are the wavelet-transformed and $\mathrm{Z}$ scale-normalized BP and SNA fluctuations, respectively).

Fourier transform analysis does not indicate localization of a particular frequency along the target signal, but provides a cumulate power of a particular frequency. In contrast, wavelet transformation does not require that the signal be stationary, and it compromises for localization of an event in both time and frequency. As indicated in this study, although spectral analysis showed a close coupling between fluctuations in BP and SNA at stimulating frequencies of from 0.1 to $0.85 \mathrm{~Hz}$ (Fig. 1BII), only the fluctuations within the frequency band of $0.25 \sim 0.4 \mathrm{~Hz}$ demonstrated a good linear fit in the time domain (Fig. 1BIV). Since our experimental system is simplified, the sole 
A

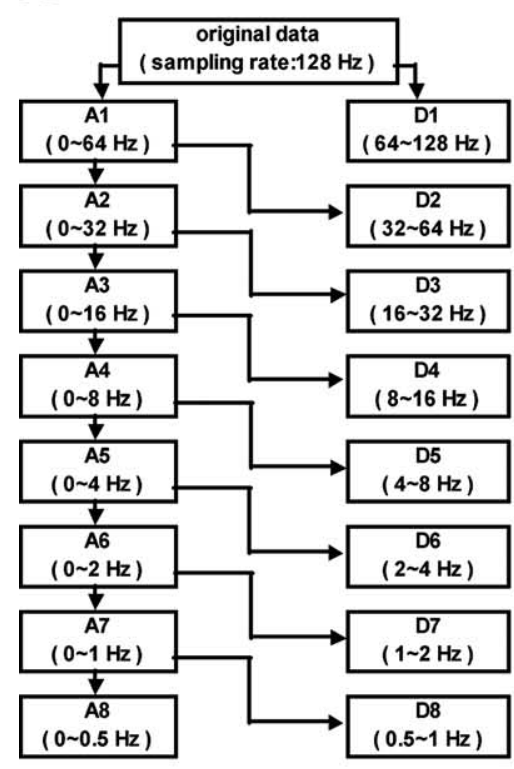

B

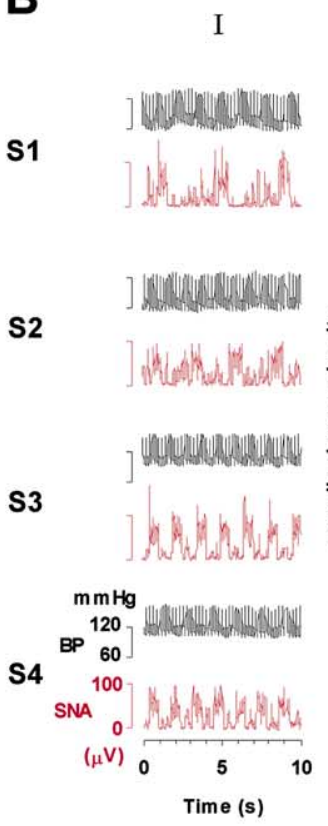

II
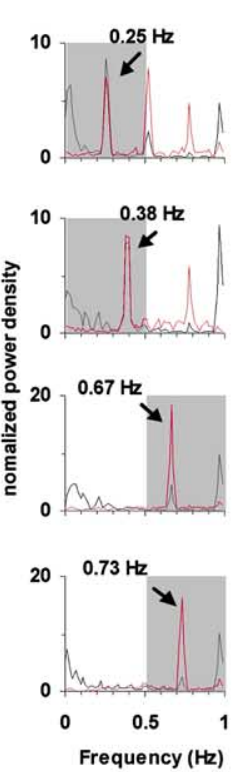

III
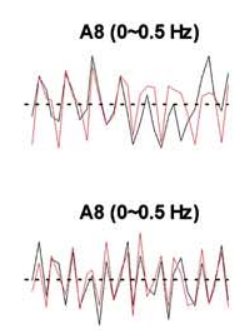

D8 $(0.5 \sim 1 \mathrm{~Hz})$

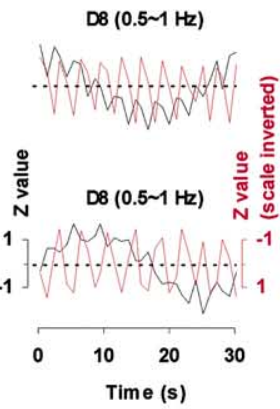

IV
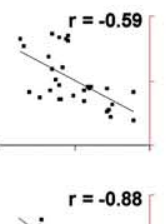

$\therefore$
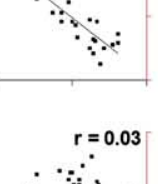
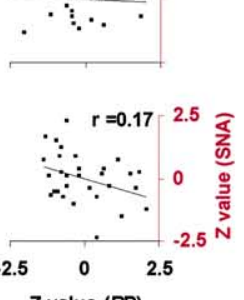

Fig. 1. Rationale for choosing the order of discrete wavelet transformation and analyzing the relationship of BP and SNA fluctuations. (A) Diagram of the frequency range of different orders of DWT. (B) Representative examples of analyzing the relationship of BP and SNA fluctuations, including their respective spectral powers and wavelet transformations during electrical stimulation of the medullary at different frequencies: S1, 0.25 Hz; S2, $0.38 \mathrm{~Hz} ; \mathrm{S} 3,0.67 \mathrm{~Hz}$; and S4, $0.73 \mathrm{~Hz}$. Column I: the original recorded traces of BP and integrated SNA. Column II: the respective spectral power of BP and SNA of Column I; a $64 \mathrm{~s}$ analysis period was used. The gray area indicates the frequency range for DWT. Notice that there is only one peak of sympathetic activity within this range. Column III: DWT of the respective BP and SNA fluctuations The order of DWT was chosen to fit the frequency range indicated in Column II. Notice that the scale of SNA is shown as being inverted. Column IV: plots of paired Z values of BP (X-axis) versus SNA (Y-axis) of each time in Column III. The regression line and the correlation coefficient are shown in each graph.

source of fluctuations of the BP could only be ascribed to changes in the vascular sympathetic activities. The results further suggested that the fluctuations transmitted from SNA to BP were uniform without being shifted by the nature of the vasculature or lagging by the sympathetic action in the frequencies centered around $0.25 \sim 0.4 \mathrm{~Hz}$.

Analysis of these fluctuations of BP and SNA by means of the transfer function also revealed similar results. The relationship between the frequency and the logarithmic magnitude of the transfer function in the low-frequency range of the three rats was also analyzed by a linear regression method. The correlation coefficient was 0.97 .
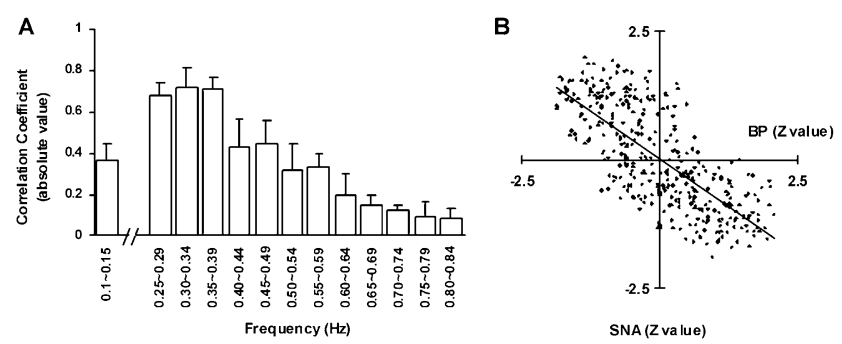

Fig. 2. Linear regression analysis of the relationship between the wavelettransformed BP and SNA of three rats. (A) The distribution histogram of the correlation coefficient. The frequency domain of $0.25 \sim 0.4 \mathrm{~Hz}$ shows a higher correlation. (B) Plots of all paired $\mathrm{Z}$ values of BP (X-axis) versus SNA (Y-axis) in the frequency range from 0.25 to $0.4 \mathrm{~Hz}$ over the course of time. The correlation coefficient of the pooled plots is 0.74 , and the formula of the regression line is $\mathrm{Y}=-0.75 \mathrm{X}$.
The X-intercept of regression line was $0.32 \mathrm{~Hz}$ (Fig. 3), which means that the fluctuations of the two signals are equal in quantity at a frequency of around $0.32 \mathrm{~Hz}$. In contrast, although coupling peaks were observed in the spectra of BP and SNA, the power densities of both were unequal quantities at frequencies lower or higher than 0.32 $\mathrm{Hz}$. For example, at around $0.6 \mathrm{~Hz}$, the transfer magnitude was about -0.5 , which means that the spectral power of BP was about $1 / 3$ the power of SNA. The unequal fluctuation might have been due to the intrinsic time delay in effector

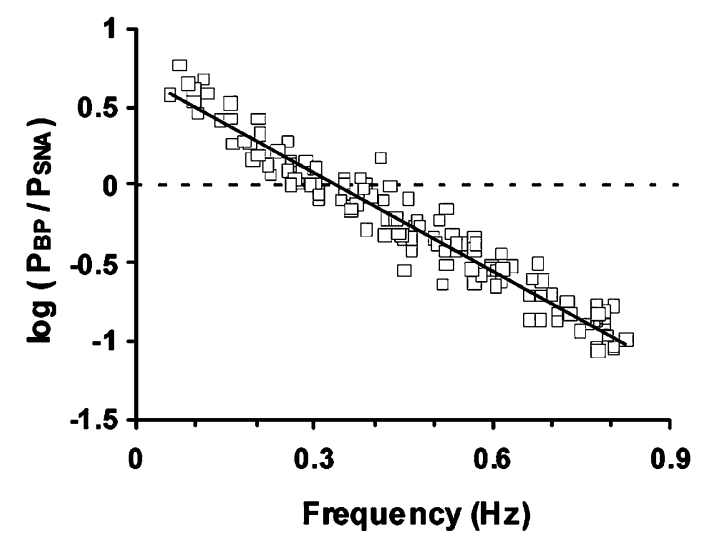

Fig. 3. Semi-logarithmic plots of the power ratio of BP and SNA in a frequency range lower than $0.85 \mathrm{~Hz}$. Data of the three rats were pooled. The correlation coefficient of the pooled plots is 0.97 , and the X-intercept of the regression line is $0.32 \mathrm{~Hz}$. 
responses to sympathetic modulation of the vasculature, while fluctuations of BP faster than $0.32 \mathrm{~Hz}$ do not entirely manifest in the vasculature but instead contribute to the mean level of vasoconstriction. These findings agree with a previous study of Brown et al. [3], who demonstrated a close coupling between fluctuations in SNA and BP at $0.4 \mathrm{~Hz}$ both in conscious and pentobarbital-anesthetized rats. Stauss and Kregel [11] also showed that transmission from peripheral sympathetic nerves to vascular smooth muscles is strongest in the frequency band from 0.2 to $0.5 \mathrm{~Hz}$ in conscious rats.

With this simplified system, we demonstrate a new possibility of exerting wavelet transformation for quantitation of sympathetic variability by BP at time domain. Although our present data support the use of low-frequency variability of BP as a quantitative index of sympathetic variability in a restricted condition, the precise utility of the relationship remains to be determined. Physiological conditions call for further study of possible complications from various control factors.

\section{References}

[1] M. Akay, Introduction: wavelet transforms in biomedical engineering, Ann. Biomed. Eng. 23 (1995) 529-530.

[2] S. Akselrod, S. Eliash, O. Oz, S. Cohen, Hemodynamic regulation in SHR: investigation by spectral analysis, Am. J. Physiol. 253 (1987) H176-H183.

[3] D.R. Brown, L.V. Brown, A. Patwardhan, D.C. Randall, Sympathetic activity and blood pressure are tightly coupled at $0.4 \mathrm{~Hz}$ in conscious rats, Am. J. Physiol. 267 (1994) R1378-R1384.

[4] C. Cerutti, M.P. Gustin, C.Z. Paultre, M. Lo, C. Julien, M. Vincent, J. Sassard, Autonomic nervous system and cardiovascular variability in rats: a spectral analysis approach, Am. J. Physiol. 261 (1991) H1292-H1299.
[5] R. Furlan, A. Porta, F. Costa, J. Tank, L. Baker, R. Schiavi, D. Robertson, A. Malliani, R. Mosqueda-Garcia, Oscillatory patterns in sympathetic neural discharge and cardiovascular variables during orthostatic stimulus, Circulation 101 (2000) 886-892.

[6] B.J.A. Janssen, J. Oosting, D.W. Slaaf, P.B. Persson, H.A.J. StruijkerBoudier, Hemodynamic basis of oscillations in systemic arterial pressure in conscious rats, Am. J. Physiol. 269 (1995) H62-H71.

[7] C. Julien, Z.Q. Zhang, C. Cerutti, C. Barrès, Hemodynamic analysis of arterial pressure oscillations in conscious rats, J. Auton. Nerv. Syst. 50 (1995) 239-252.

[8] S. Perlini, F. Giangregorio, M. Coco, A. Radaelli, P.L. Soldà, L. Bernardi, A.U. Ferrari, Autonomic and ventilatory components of heart rate and blood pressure variability in freely behaving rats, Am. J. Physiol. 269 (1995) H1729-H1734.

[9] P.B. Persson, H. Stauss, O. Chung, U. Wittmann, T. Unger, Spectrum analysis of sympathetic nerve activity and blood pressure in conscious rats, Am. J. Physiol. 263 (1992) H1348-H1355.

[10] A. Radaelli, L. Bernardi, F. Valle, S. Leuzzi, F. Salvucci, L. Pedrotti, E. Marchesi, G. Finardi, P. Sleight, Cardiovascular autonomic modulation in essential hypertension: effect of tilting, Hypertension 24 (1994) 556-563.

[11] H.M. Stauss, K.C. Kregel, Frequency response characteristic of sympathetic-mediated vasomotor waves in conscious rats, Am. J. Physiol. 271 (1996) H1416-H1422.

[12] H.M. Stauss, R. Mrowka, B. Nafz, A. Patzak, T. Unger, P.B. Persson, Does low frequency power of arterial blood pressure reflect sympathetic tone? J. Auton. Nerv. Syst. 54 (1995) 145-154.

[13] Task Force of the European Society of Cardiology and the North American Society of Pacing and Electrophysiology, Heart rate variability, Standards of measurement, physiological interpretation, and clinical use, Circulation 93 (1996) 1043-1065.

[14] M.L. Tsai, L.W. Chu, C.Y. Chai, C.T. Yen, Frequency dependent sympathetic modulation of vasomotor tone in the anesthetized rat, Neurosci. Lett. 221 (1997) 109-112.

[15] M.L. Tsai, F.Z. Shaw, C.T. Yen, The quantitative relationship between the fluctuations of blood pressure and sympathetic nerve activity in pentobarbital anesthetized rats, Neurosci. Lett. 263 (1999) 85-88. 